\title{
Advantages of Communication in Double Three-Phase Surface Permanent Magnet Machines Fed by Independent Inverters
}

\author{
G. Sala, M. Mengoni, A. Tani \\ Dept. of Electrical, Electronic, and Information Engineering \\ University of Bologna, Bologna, IT \\ giacomo.sala5@unibo.it \\ A. Galassini, M. Degano \\ Power Electronics, Machines and Control (PEMC) Group \\ University of Nottingham, Nottingham, UK
}

\section{Keywords}

Analytical Model, Multi Three-Phase Drives, Surface Permanent Magnet Machines, Control and Communication.

\begin{abstract}
This paper deals with a double three-phase Surface Permanent Magnet (SPM) drive where two independent three-phase inverters are supplying the same electrical machine. This work highlights how the magnetic coupling of the two windings affects the control performance. A new control strategy based on a low bandwidth communication, aiming to improve the performance of the system, is presented and validated by numerical simulations.
\end{abstract}

\section{Introduction}

The development of new materials and related technologies is allowing to design electrical drives with higher levels of power, performance and reliability. A further step for the development of electrical drives has been reached by the multiphase solutions, where the overall power of electrical machine is split in a higher number of phases and related converter legs [1,2]. Among the multiphase layouts, the multi three-phase offers advantages in terms of simplicity and modularity of the converter design, which can be obtained by conventional three-phase inverters.

In the literature of multi three-phase drives, there are different approaches to the definition of the machine model and the related customized control. Among these, it is worth to highlight the multi space vector approach (centralised) and the redundant three-phase one (distributed) [3]. In the former the machine is considered as an unique device, described by a set equations related to the control of different field harmonics of the machine $[4,5,6]$, whereas in the latter each three-phase system is considered by its representing equations, and the coupling with the others is considered as a disturbance or neglected [7, 8]. While the first approach is essential to properly control more harmonics of the airgap magnetic field, the second allows for having two completely independent controllers for each three-phase system (resulting in an interesting approach for the development of systems with high reliability requirements). This paper aims to highlight the effects related to the use of independent controllers in terms of control design and performance. A possible intermediate solution, based on a relatively low bandwidth communication among the two controllers, is introduced. Finally, numerical simulations of a double three-phase SPM machine drive are presented to validate the advantages of the proposed communication.

\section{Analytical Model: Double Three-Phase SPM Machine}

The analysed drive is schematically represented in Fig. 1. The two inverters in Fig. 1(a) are supplying two three-phase windings of the six-phase SPM machine, shifted by 30 electrical degrees as shown in 


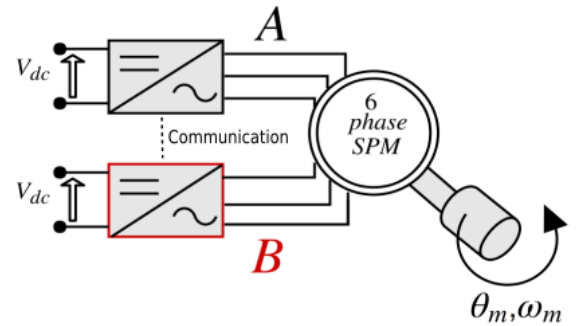

a)

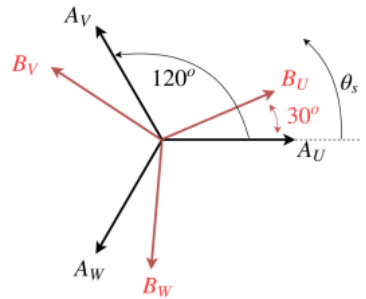

b)

Fig. 1: Layout of a double three-phase drive $(a)$ and distribution of the magnetic axes of the double threephase machine $(b)$. The phase shift between the two three-phase subsystems is 30 electrical degrees.

Fig. 1(b). The electrical quantities (currents and voltages) of each three-phase winding $(A, B)$ can be represented in terms of Space Vectors (SVs) by the Clarke transformation:

$$
\bar{y}_{A, \rho}^{A}=\frac{2}{3}\left(x_{A, U}+x_{A, V} e^{j \rho \frac{2 \pi}{3}}+x_{A, W} e^{j \rho \frac{4 \pi}{3}}\right), \bar{y}_{B, \rho}^{B}=\frac{2}{3}\left(x_{B, U}+x_{B, V} e^{j \rho \frac{2 \pi}{3}}+x_{B, W} e^{j \rho \frac{4 \pi}{3}}\right), \rho=0,1,
$$

where $j$ is the unity imaginary number, $\rho$ is the vector space of the SV representation, and the superscripts $\xi$ are used to specify the reference frame where the quantities are defined. For example, ${ }^{A}$ and ${ }^{B}$ in (1) refer to the reference frame centered with the magnetic axis of the $U$ phase of the system A (chosen also as stator reference frame) and B (shifted by 30 electrical degrees from A) respectively.

For an electrical machine, the complex numbers $\bar{y}_{A, 1}^{\xi}$ and $\bar{y}_{B, 1}^{\xi}$ in (1) are the $1^{\text {st }} \mathrm{SVs}$ in the respective $\alpha_{\xi}-\beta_{\xi}$ complex planes, while $y_{A, 0}$ and $y_{B, 0}$ represent the zero sequence components. Assuming that the two three-phase windings are independently star connected, the zero sequence currents $i_{A, 0}$ and $i_{B, 0}$ are set to zero, and the machine currents are completely determined by the current space vectors $\bar{i}_{A, 1}^{A}$ and $\bar{i}_{B, 1}^{B}$. The control of these current SVs is completely associated to the related voltage SVs $\bar{v}_{A, 1}^{A}$ and $\bar{v}_{B, 1}^{B}$ by the following relationships:

$$
\bar{v}_{A, 1}^{A}=R_{S} \bar{i}_{A, 1}^{A}+\frac{d \bar{\phi}_{A, 1}^{A}}{d t}, \quad \bar{v}_{B, 1}^{B} \quad=R_{S} \bar{i}_{B, 1}^{B}+\frac{d \bar{\phi}_{B, 1}^{B}}{d t}
$$

with $R_{S}$ the phase resistance, $\bar{\phi}_{A, 1}^{A}$ and $\bar{\phi}_{B, 1}^{B}$ the SVs of the linked fluxes of the respective three-phase winding. Introducing the angle $\vartheta$ representing the rotor electrical position with respect to the stator reference frame $\left(\vartheta=p \vartheta_{m}\right)$, the linked flux space vectors $\left(\bar{\phi}_{A, 1}^{A}\right.$ and $\left.\bar{\phi}_{B, 1}^{B}=\bar{\phi}_{B, 1}^{A} e^{-j \vartheta_{B}}\right)$ can be respectively rewritten as:

$$
\begin{aligned}
& \bar{\phi}_{A, 1}^{A}=L \bar{i}_{A, 1}^{A}+M \bar{i}_{B, 1}^{B} e^{j \vartheta_{B}}+\sum_{\substack{h=0 \\
\rho=6 h+1}}^{\infty} \bar{\phi}_{P M, \rho} e^{j \rho \vartheta}+\sum_{\substack{h=1 \\
\rho=6 h-1}}^{\infty} \bar{\phi}_{P M, \rho} e^{-j \rho \vartheta}, \\
& \bar{\phi}_{B, 1}^{B}=L \bar{i}_{B, 1}^{B}+M \bar{i}_{A, 1}^{A} e^{-j \vartheta_{B}}+\sum_{\substack{h=0 \\
\rho=6 h+1}}^{\infty} \bar{\phi}_{P M, \rho} e^{j \rho \vartheta} e^{-j \rho \vartheta_{B}}+\sum_{\substack{h=1 \\
\rho=6 h-1}}^{\infty} \bar{\phi}_{P M, \rho} e^{-j \rho \vartheta} e^{j \rho \vartheta_{B}},
\end{aligned}
$$

with $L$ and $M$ the equivalent self and mutual inductances of the two windings in the SV representation, and $\bar{\phi}_{P M, \rho}$ the SV which takes into account for the $\rho t h$ harmonic of the permanent magnet flux linked with each system. For the purpose of describing the machine behaviour and defining a suitable control algorithm (3) can be rewritten considering all the SV in the $d-q$ rotor reference frame, oriented with the north pole of the $1^{s t}$ harmonic of the magnetic field of the rotor $\left(\bar{y}_{A, 1}=y_{A, d 1}+j y_{A, q 1}=\bar{y}_{A, 1}^{A} e^{-j \vartheta}\right.$ and $\left.\bar{y}_{B, 1}=y_{B, d 1}+j y_{B, q 1}=\bar{y}_{B, 1}^{B} e^{-j\left(\vartheta-\vartheta_{B}\right)}\right)$, and highlighting the main contribution of the permanent magnet 
back electromotive force (bemf) as:

$$
\begin{aligned}
& \bar{\phi}_{A, 1}=L \bar{i}_{A, 1}+\phi_{P M, 1}+M \bar{i}_{B, 1}+\sum_{\substack{h=1 \\
\rho=6 h \pm 1}}^{\infty} \phi_{P M, \rho} e^{ \pm j \rho \vartheta-j \vartheta}, \\
& \bar{\phi}_{B, 1}=L \bar{i}_{B, 1}+\phi_{P M, 1}+M \bar{i}_{A, 1}+\sum_{\substack{h=1 \\
\rho=6 h \pm 1}}^{\infty} \phi_{P M, \rho} e^{j \rho\left( \pm \vartheta \mp \vartheta_{B}\right)-j \vartheta+j \vartheta_{B}} .
\end{aligned}
$$

Equation (4) can be introduced in (3) to obtain the voltage SV equations in the rotor reference frame:

$$
\begin{aligned}
\bar{v}_{A, 1} & =R_{S} \bar{i}_{A, 1}+L \frac{d \bar{i}_{A, 1}}{d t}+j \omega L \bar{i}_{A, 1}+j \omega \phi_{P M, 1}+ \\
& +M \frac{d \bar{i}_{B, 1}}{d t}+j \omega M \bar{i}_{B, 1}+\sum_{\substack{h=1 \\
\rho=6 h \pm 1}}^{\infty} \pm j \rho \omega \phi_{P M, \rho} e^{j(-1 \pm \rho) \vartheta} \\
\bar{v}_{B, 1} & =R_{S} \bar{i}_{B, 1}+L \frac{d \bar{i}_{B, 1}}{d t}+j \omega L \bar{i}_{B, 1}+j \omega \phi_{P M, 1}+ \\
& +M \frac{d \bar{i}_{A, 1}}{d t}+j \omega M \bar{i}_{A, 1}+\sum_{\substack{h=1 \\
\rho=6 h \pm 1}}^{\infty} \pm j \rho \omega \phi_{P M, \rho} e^{j\left[(-1 \pm \rho) \vartheta+(1 \mp \rho) \vartheta_{B}\right]}
\end{aligned}
$$

with $\omega$ the angular speed of the machine in electrical radians $\left(\omega=p \omega_{m}=p \frac{d \vartheta_{m}}{d t}\right)$. Finally, the torque transferred from the stator to the rotor $\left(T_{6 p h}\right)$ can be evaluated considering separately the main torque contribution and the torque ripple as:

$$
T_{6 p h}=\frac{3}{2} p \phi_{P M, 1}\left(i_{A, q 1}+i_{B, q 1}\right)+\frac{3}{2} \sum_{\substack{h=1 \\ \rho=6 h \pm 1}}^{\infty} \Re\left\{ \pm \rho p \phi_{P M, \rho} e^{j(-1 \pm \rho) \vartheta}\left[i_{A, q 1}+i_{B, q 1} e^{j(1 \mp \rho) \vartheta_{B}}\right]\right\},
$$

with $p$ the number of pole pairs.

It is worth noticing that if the two converters are controlled with the same reference torque $\left(i_{A, q 1}=i_{B, q 1}=\right.$ $\left.i_{q 1}\right)$, the contribution to the torque ripple expected by all the harmonics of the permanent magnet field distribution related to odd $h$ values $(\rho=6 h \pm 1)$ are theoretically eliminated (in particular the $5^{\text {th }}$ and the $7^{\text {th }}$, related to $h=1$ ):

$$
T_{6 p h, \text { balanced }}=\frac{6}{2} p \phi_{P M, 1} i_{q 1}+\frac{6}{2} i_{q 1} \sum_{z=1}^{\infty} \pm(12 z \pm 1) p \phi_{P M,(12 z \pm 1)} \cos (12 z \vartheta)
$$

It results that, even if not the main investigation of this work, the independent FOC of the two systems is not the optimal control solution to compensate the disturbance induced in the machine currents by the higher order harmonics of the bemf caused by the magnets, but the reference currents in the conventional operation (equal torque sharing of $\mathrm{A}$ and $\mathrm{B}$ ) are still the optimized ones for the minimization of the machine torque ripples until the two inverters are controlling the same torque $\left(i_{q 1}\right)$. The control of the double three-phase machine presented in the next section is based on the analysis of (5).

\section{Independent Controls and Communication}

Under the assumptions of the model, if the two three-phase windings were magnetically decoupled and the distribution of the flux density in the airgap generated by the permanent magnets were supposed sinusoidal the voltage equations (5) of the two inverters could be simply defined as:

$$
\begin{aligned}
& \bar{v}_{A, 1}^{\prime}=R_{S} \bar{i}_{A, 1}+L \frac{d \bar{i}_{A, 1}}{d t}+j \omega L \bar{i}_{A, 1}+j \omega \phi_{P M, 1}, \\
& \bar{v}_{B, 1}^{\prime}=R_{S} \bar{i}_{B, 1}+L \frac{d \bar{i}_{B, 1}}{d t}+j \omega L \bar{i}_{B, 1}+j \omega \phi_{P M, 1} .
\end{aligned}
$$


To consider for the mutual coupling of the two systems the contributions that have be added are the followings:

$$
\bar{v}_{A, 1}^{\prime \prime}=M \frac{d \bar{i}_{B, 1}}{d t}+j \omega M \bar{i}_{B, 1}, \quad \bar{v}_{B, 1}^{\prime \prime} \quad=M \frac{d \bar{i}_{A, 1}}{d t}+j \omega M \bar{i}_{A, 1},
$$

whereas the effect of the higher order harmonics of the permanent magnets can be included as:

$$
\bar{v}_{A, 1}^{\prime \prime \prime}=\sum_{\substack{h=1 \\ \rho=6 h \pm 1}}^{\infty} \pm j \rho \omega \phi_{P M, \rho} e^{j(-1 \pm \rho) \vartheta}, \quad \bar{v}_{B, 1}^{\prime \prime \prime} \quad=\sum_{\substack{h=1 \\ \rho=6 h \pm 1}}^{\infty} \pm j \rho \omega \phi_{P M, \rho} e^{j\left[(-1 \pm \rho) \vartheta+(1 \mp \rho) \vartheta_{B}\right]} .
$$

Equations (8), (9) and (10) make possible to define the voltage equations (5) in the shape of $\bar{v}_{A, 1}=$ $\bar{v}_{A, 1}^{\prime}+\bar{v}_{A, 1}^{\prime \prime}+\bar{v}_{A, 1}^{\prime \prime \prime}$ and $\bar{v}_{B, 1}=\bar{v}_{B, 1}^{\prime}+\bar{v}_{B, 1}^{\prime \prime}+\bar{v}_{B, 1}^{\prime \prime \prime}$. Because the third term in the voltage equations $\left(\bar{v}_{A, 1}^{\prime \prime \prime}\right.$ and $\left.\bar{v}_{B, 1}^{\prime \prime \prime}\right)$ is not related to the magnetic coupling of the two systems, it is not considered and analysed in the definition of proposed control. Instead, the focus of the analysis, for the control design, is on the second terms $\bar{v}_{A, 1}^{\prime \prime}$ and $\bar{v}_{B, 1}^{\prime \prime}$. In particular, the aim is to define a minimum amount of data, shared with a reasonably slow communication, that can be transferred from the controller of one inverter to the controller of the other and which allows the control performance to be improved keeping acceptable stability margins. Indeed, it is known that for independent controllers of a multiphase drive the bandwidth of the each controller is strictly related with the effect that a current change in one of the other inverters has on its winding [7]. When thus effect, i.e. the mutual coupling of the three-phase windings, is taken into account in the control design assuming a slow communication among the inverters, the terms in (9) might significantly change with the time before the other system receives them. On the other hand, even assuming an instantaneous communication, the controller of each inverter can compensate the bemfs induced by the phase currents of the other winding, but the dynamic of this compensation might result in a control instability.

In order to improve the control performance, the proposed control algorithm is based on the tuning of each system as if there were no magnetic coupling with the other winding, and a feedforward compensation of the bemfs generated by the magnetic coupling is defined. To allow reducing the frequency of the data transmission between the controllers, whereas the traditional compensation of the dynamic bemfs $j \omega L \bar{i}_{A, 1}$ and $j \omega L \bar{i}_{B, 1}$ is implemented with the measured currents on each inverter itself, all the feedforward compensations of the mutual couplings in (9) are implemented by means of a filtered value of the reference currents rather than the measured ones. The block diagram of the proposed control algorithm is shown in Fig. 2. The system is assumed to be controlled in terms of torque contributions from the two inverters: whereas the $d$-axis currents $i_{A, d 1}$ and $i_{B, d 1}$ are maintained zero, the $q$-axis contributions are evaluated from the respective torque demands via the proportional regulators $\mathrm{P}$ equally tuned with a gain equal to the torque constant $G(P)=\frac{2}{3 p \phi_{P M, 1}}$.

Each system receives the measurement of its currents and the rotor position in order to command the suitable voltages, from the related inverter, needed for the implementation of the current control. The reference voltages are obtained as sum of the PIs $(a)$ and $(b)$ outputs with the feedforward compensation of the magnets bemfs, self dynamic bemfs $\left(j \omega L \bar{i}_{A, 1}\right.$ and $\left.j \omega L \bar{i}_{B, 1}\right)$, and mutual coupling bemfs among the windings via the implemented communication (COMM).

The control diagram and the simulations are presented for a torque ( $i_{q}$ current) control, for simplicity and to neglect the effects of the speed control on the performance of the current regulation.

\section{Tuning of the PI Regulators and Stability Analysis}

In order to perform the tuning and stability analysis three approaches have been considered. In agreement with the considerations presented in [3], the stability analysis can be performed analysing the closed loop transfer functions of the overall system modelled with the Vector Space Decomposition (VSD) theory $[9,10,11]$. In particular, as well described in [12] it is possible to introduce a current vector $\bar{i}_{1}^{S}$ related to the torque control, and a current vector $\bar{i}_{5}^{S}$ related to the unbalance in the currents of the two supply channels (the multiphase SVs are defined in the stator reference frame $S, S=A$ ). The space vectors of the 


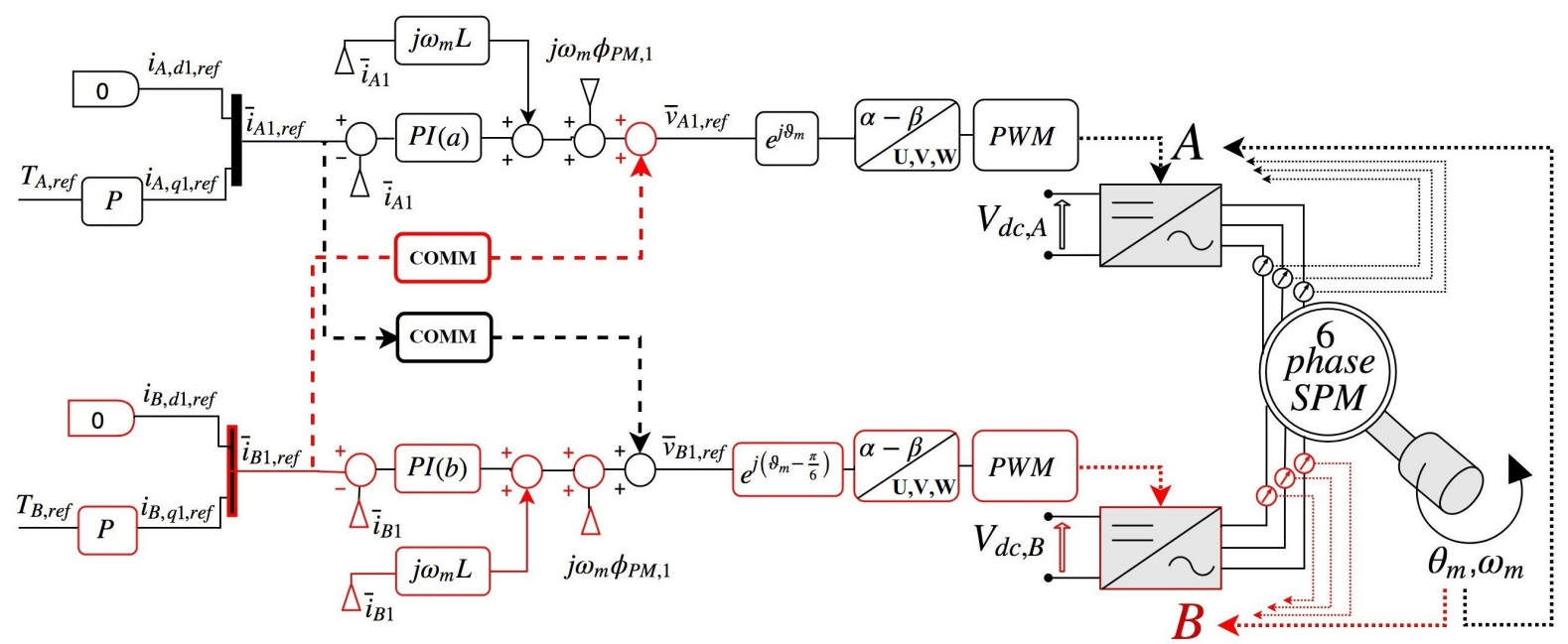

Fig. 2: Control diagram of the double three-phase drive.

machine quantities (currents, voltages and linked fluxes) can be represented in many ways, in particular the general transformation for a dual three-phase drive (11) can be defined.

$$
\bar{y}_{\rho}^{S}=\frac{2}{6}\left(x_{A, U}+x_{A, V} e^{j \rho \frac{4 \pi}{6}}+x_{A, W} e^{j \rho \frac{8 \pi}{6}}\right)+\frac{2}{6}\left(x_{B, U} e^{j \rho \frac{\pi}{6}}+x_{B, V} e^{j \rho \frac{5 \pi}{6}}+x_{B, W} e^{j \rho \frac{9 \pi}{6}}\right), \rho=0,1, \ldots \infty .
$$

Applying (11) to the machine currents and voltages for the spaces 1 and $5(\rho=1,5)$, and introducing the three-phase space vectors (1) represented in the rotor reference frame, the torque and the current unbalance SVs (1 and 5) can be defined as:

$$
\bar{i}_{1}=\frac{\bar{i}_{A, 1}+\bar{i}_{B, 1}}{2}, \quad \bar{i}_{5}=\frac{\bar{i}_{A, 1}^{*}-\bar{i}_{B, 1}^{*}}{2}, \quad \bar{v}_{1}=\frac{\bar{v}_{A, 1}+\bar{v}_{B, 1}}{2}, \quad \bar{v}_{5}=\frac{\bar{v}_{A, 1}^{*}-\bar{v}_{B, 1}^{*}}{2},
$$

and the machine equations (5) can be rewritten in the $1^{s t}$ and $5^{t h} d-q$ planes as:

$$
\begin{aligned}
& \bar{v}_{1}=R_{S} \bar{i}_{1}+(L+M) \frac{d \bar{i}_{1}}{d t}+j \omega(L+M) \bar{i}_{1}+j \omega \phi_{P M, 1}+\sum_{\substack{h=1 \\
\rho=12 h \pm 1}}^{\infty} \pm j \rho \omega \phi_{P M, \rho} e^{j(-1 \pm \rho) \vartheta}, \\
& \bar{v}_{5}=R_{S} \bar{i}_{5}+(L-M) \frac{d \bar{i}_{5}}{d t}+j \omega(L-M) \bar{i}_{5}+\sum_{\substack{h=1 \\
\rho=(12 h-6) \pm 1}}^{\infty} \pm j \rho \omega \phi_{P M, \rho} e^{j(-1 \pm \rho) \vartheta} .
\end{aligned}
$$

Assuming that the two-individual current controls, of inverter A and B respectively, have to be equally tuned, the current vector PI regulators of the two inverters have the following representing equations in the Laplace domain:

$$
\bar{V}_{A, 1, r e f}=\left(k_{p}+\frac{k_{i}}{s}\right)\left(\bar{i}_{A, 1, \text { ref }}-\bar{i}_{A, 1, \text { mea }}\right), \quad \bar{V}_{B, 1, \text { ref }}=\left(k_{p}+\frac{k_{i}}{s}\right)\left(\bar{i}_{B, 1, \text { ref }}-\bar{i}_{B, 1, \text { mea }}\right),
$$

with $\bar{y}_{A(B), 1, r e f}$ and $\bar{y}_{A(B), 1, \text { mea }}$ the SVs of the inverter reference (ref) and measured (mea) quantities. From the inverter PI representation, the transfer functions between the error of the current SVs and the reference voltage SVs can be obtained substituting (14) in (12) as [3]:

$$
\bar{V}_{1, \text { ref }}=\left(k_{p}+\frac{k_{i}}{s}\right)\left(\bar{i}_{1, \text { ref }}-\bar{i}_{1, \text { mea }}\right), \quad \bar{V}_{5, \text { ref }}=\left(k_{p}+\frac{k_{i}}{s}\right)\left(\bar{i}_{5, \text { ref }}-\bar{i}_{5, \text { mea }}\right) .
$$

Therefore, among the possible strategies for the tuning of the PI regulators there are three that seems to be of interest:

a) tuning for the maximum torque performance (tracking of $\bar{i}_{1, \text { ref }}$ ); 
b) tuning for the minimum current distortion caused by the currents unbalances $\bar{i}_{A, 1}-\bar{i}_{B, 1}$ (tracking of $\bar{i}_{5, r e f}$ );

c) tuning each system as if there were not mutual coupling of the inverters (proposed, tracking of $\left.\bar{i}_{A(B), 1, r e f}\right)$.

Strategy (a) allows for the maximum torque performance, but the tuning of the PI regulators based on the transfer function of the space vectors in the plane 1 in (12) requires a significant reduction of the gain of the PIs to avoid control instabilities when $(L+M) /(L-M)$ is high (as in the analysed case study, with $(L+M) /(L-M)=13)$ [3]. The control instabilities rise from the closed loop response of the plant described in the plane 5 by (13), where the time constant $(L-M) / R_{S}$ is much smaller than the one in the plane $1(L+M) / R_{S}$.

Strategy $(b)$ allows a smooth tracking of the reference currents avoiding recirculating currents (significant opposite currents in the two inverters caused by a bouncing of the current disturbance among the two inverter PI regulators), but leads to a poor dynamic of the current regulators for the torque generation.

Strategy $(c)$ bases the current control tuning on the methodologies used in standard three-phase drives assuming that one system is not acting on the other, resulting in a simple implementation and understanding. This solution is an intermediate one between the $(a)$ and $(b)$ regulator tuning techniques, and it is also a reasonable alternative solution to the controls for dual three-phase drives where power sharing or fault tolerant algorithms are desired $[8,13]$. As for case $(a)$, also in this scenario a reduction of the regulators gain is needed to avoid instabilities caused by the control dynamic of the plant mapped on the $5^{\text {th }}$ plane.

Fig. 3(a-c) show the closed loop Bode and pole root locus diagrams of the controlled system in terms of space vector planes 1 (VSD-1) and 5 (VSD-5), and inverter (INV-A(B)) when the PIs are tuned in zero-pole cancellation with the considered plant $\left((L+M) / R_{S},(L-M) / R_{S}\right.$ and $L / R_{S}$, respectively) for the three analysed tuning strategies $(a, b, c)$. Whereas at the top of Fig. $3(a-c)$ are presented the resulting diagrams for the initial tuning, at the bottom of Fig. $3(a)$ and Fig. $3(c)$ also the diagrams in case of reduced gain of the PI regulators (needed to ensure the stability of the control system) are displayed. The significant reduction of the gain of the PI regulators, required to keep a robust control algorithm of the overall system, directly affects the dynamic performance of the control decreasing the bandwidth of the closed loop current response. For the stability analysis, the delay introduced by the DSP and the inverter $\left(e^{-T_{d} s}\right.$, with $\left.T_{d}=150 \mu s\right)$ has been introduced in the representation of the transfer functions of the control plants by means of a second-order Padé approximation.

The pole root loci in Fig. 3(a) and 3(c) highlight the presence of two complex conjugate poles with a negative real part in the closed loop transfer function of the system mapped in the plane 5 . The reduction of the regulators gain of a factor of 10 and 6, respectively for case $(a)$ and $(c)$, brings the critical closed loop poles in the left hand side of the image axis and, in particular, the dumping of the obtained transfer function in the $5^{\text {th }}$ plane becomes acceptable. On the other hand, the tuning based on the plant mapped in the $5^{\text {th }}$ plane (case $b$ ) leads to a stable system, but with both a low bandwidth and resonances in the closed loop responses mapped in the $1^{s t}$ plane and inverter plant.

From the Bode diagrams and the pole root loci, the tuning strategy based on the single inverter plant has been adopted. The simulation results of the system control are presented in the next section, and compared under different control implementations of the investigated feedforward compensations.

\section{Simulation Results}

The simulation of the control has been implemented using the software Matlab-Simulink. The machine parameters, provided in Table I have been evaluated from finite element simulations of the six phase machine with the Altair software Flux. The higher order harmonics of the bemf have been finally removed from the machine model in order to better appreciate the effects of the proposed control algorithm. Fig. 4 presents the simulation results for the machine rotating at rated speed $(1500 \mathrm{rpm})$ with inverter A and B independently controlled in torque ( $i_{q}$ current). The simulation aims to evaluate the control performance when the two inverters have the same torque demand, when one inverter (B) is operating with an oppo- 

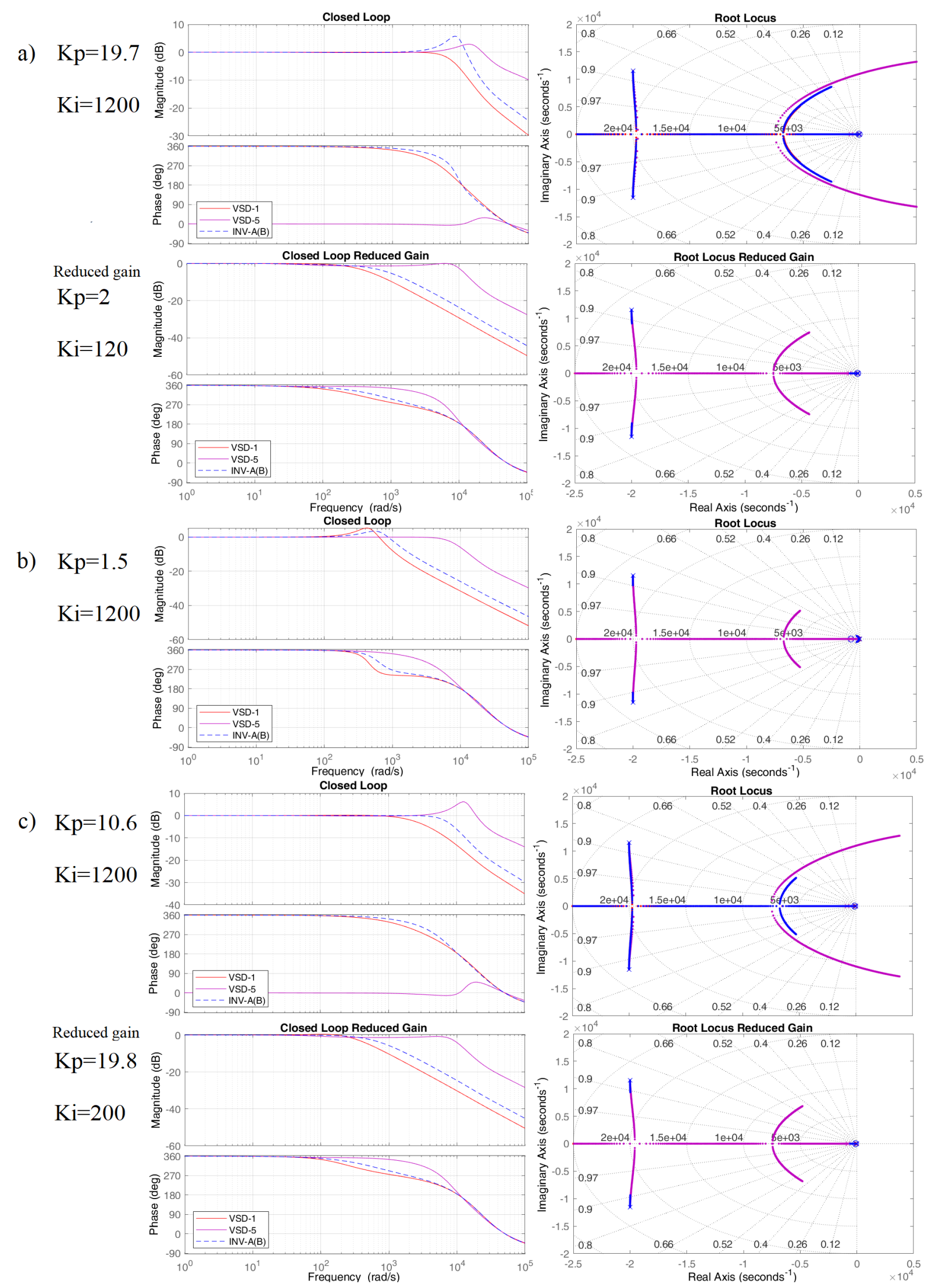

Fig. 3: Bode diagrams and pole root locus of the of the current control in terms of space 1 (red), space 5 (magenta) and inverter (dashed blue). The diagrams result from the tuning strategies based on: space 1 $(a)$, space $5(b)$ and inverter plant $(c)$. 
Table I: Machine Parameters

\begin{tabular}{l|l} 
Parameter & Value \\
\hline \hline Pole pair number $(\mathrm{p})$ & 2 \\
Phase resistance $\left(R_{S}\right)$ & $0.36[\Omega]$ \\
Self inductance $(L)$ & $3.19[\mathrm{mH}]$ \\
Mutual inductance $(M)$ & $2.73[\mathrm{mH}]$ \\
Rated torque $\left(T_{\text {rated }}\right)$ & $25[\mathrm{Nm}]$ \\
Rated speed $\left(n_{m}\right)$ & $1500[\mathrm{rpm}]$
\end{tabular}
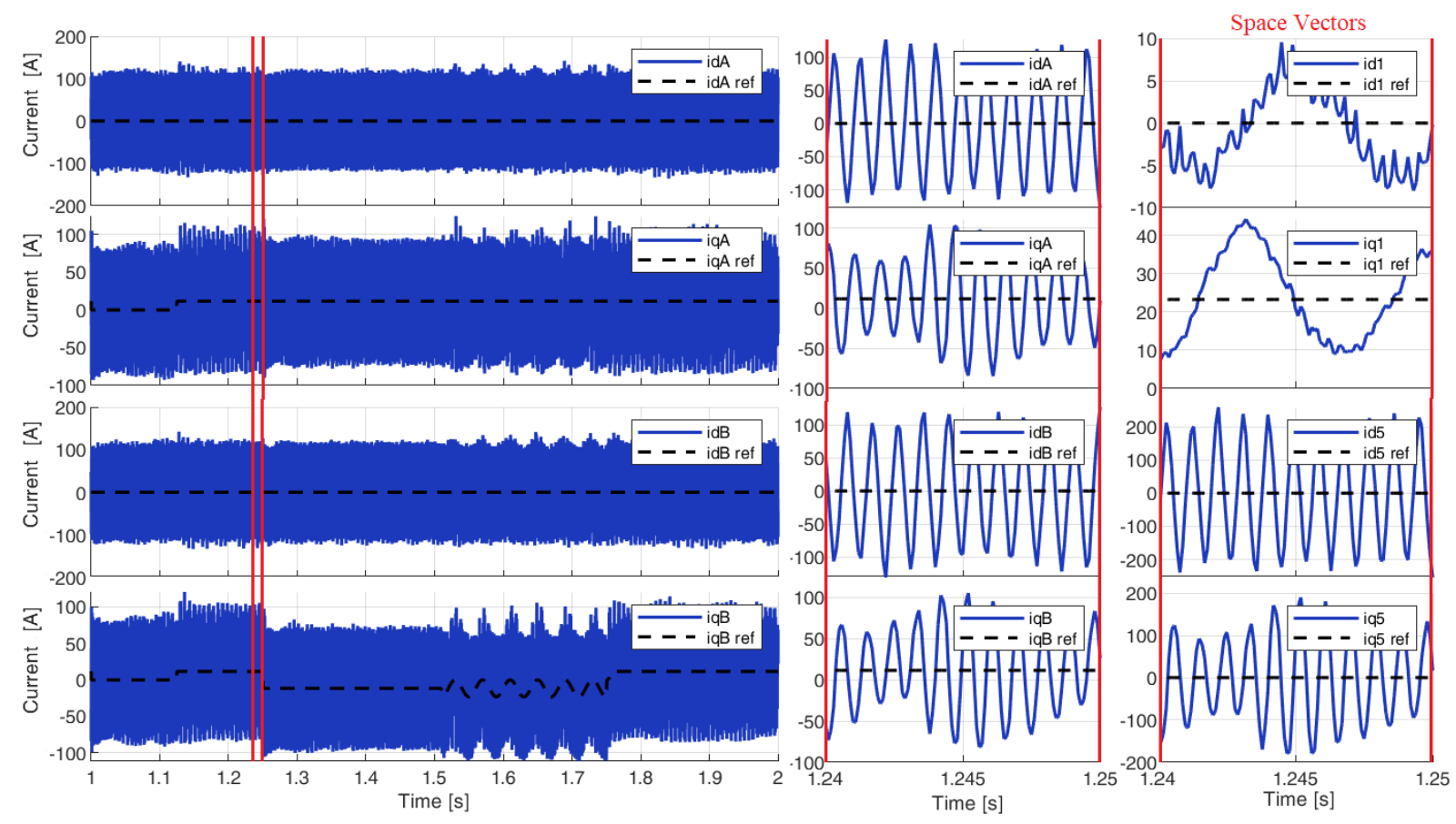

Fig. 4: Simulation of the control system with conventional tuning of the two PI regulators neglecting the effect of the other inverter.

site reference torque (power sharing), and in case of a superimposed sinusoidal reference torque request at the mechanical frequency $(25 \mathrm{~Hz})$ on one inverter (B). This working operation is cyclically repeated every second of the presented simulations.

In Fig. 4 are displayed the simulation results with the PI regulators tuned considering each inverter separately (strategy $(c)$ ) according to the conventional methodologies, i.e., neglecting the existence of the other inverter. Fig. 4 highlights how the controllers of the two inverters interact causing significant circulating currents. The current SV components, shown at the right hand side of the figure, emphasise the really poor dynamic of the equivalent reference current $\bar{i}_{5, \text { ref }}$ which result in a response of the system close to an instability.

On the opposite, the reduction of the gain (the gain has been reduced by a factor 6) allows for a stable control of the system also when the reference current of one inverter is opposite and with a significant superimposed sinusoidal reference value at the mechanical frequency. This statement is validated by the simulation results of Fig. 5, where the gain of the PI regulators has been reduced and various control techniques are implemented. In particular, the tuning of the PI regulators and the machine working operation are maintained equal in each working cycle (lasting for $1 \mathrm{~s}$ ), and the following feedforward compensations are implemented:

i) none;

ii) permanent magnet bemf $\left(j \omega \phi_{P M, 1}\right)$ and self dynamic bemf $\left(j \omega L \bar{i}_{A(B), 1, \text { mea }}\right)$; 

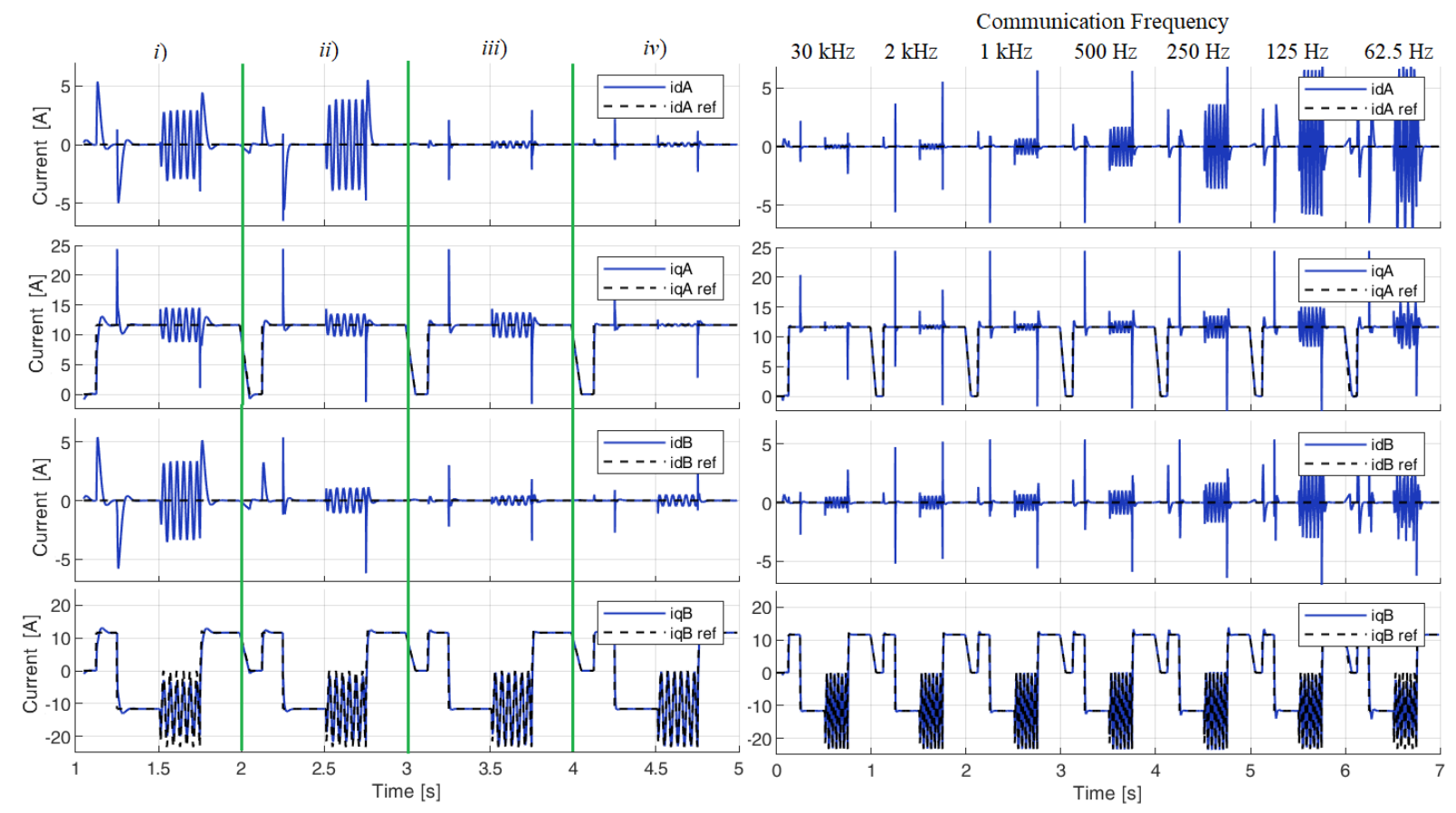

Fig. 5: Simulation of the control system with conventional tuning of the two PI regulators, reduction of the gain to reach a stable control system, and feedforward compensations. On the right hand side are shown the results with the optimal feedforward compensation $i v$ introducing a communication delay (from negligible up to a communication frequency of $62.5 \mathrm{~Hz}$ ).

iii) permanent magnet bemf ( $\left.j \omega \phi_{P M, 1}\right)$, self dynamic bemf $\left(j \omega L \bar{i}_{A(B), 1, \text { mea }}\right)$, and only the mutual coupling bemf contribution $j \omega M \bar{i}_{B(A), 1, \text { ref }}$;

iv) permanent magnet bemf $\left(j \omega \phi_{P M, 1}\right)$, self dynamic bemf $\left(j \omega L \bar{i}_{A(B), 1}\right)$, and the overall mutual coupling bemf contribution $j \omega M \bar{i}_{B(A), 1, \text { ref }}+M \frac{d \bar{i}_{B(A), 1, \text { ref }}}{d t}$.

Focusing on the various feedforward compensations, when a sinusoidal $i_{q}$ current is commanded to the inverter B, the currents of inverter B in Fig. 5(ii) are significantly improved when compared with the ones in Fig. 5(i), while there are not significant improvements on the current ripples of inverter A. Therefore, as expected, the compensation of the magnets bemf $\left(j \omega \phi_{P M, 1}\right)$ and the self dynamic bemf $\left(j \omega L \bar{i}_{A(B), 1, m e a}\right)$ improves the performance of the tracking of the reference currents of each inverter, but without advantages on the reduction of the current ripples caused by the mutual coupling of the two systems.

On the other hand, the additional compensation of the mutual couplings $j \omega M \bar{i}_{B(A), 1, \text { ref }}$ in Fig. $5(i i i)$ and finally also $M \frac{d \bar{d}_{B(A), 1, \text { ref }}}{d t}$ in Fig. 5(iv) enhance both the reduction of the currents induced by the mutual bemfs on the inverter A (who is affected by the currents of inverter B as a disturbance) and, indirectly, also the tracking of the sinusoidal $q$-axis current from the inverter supposed to control it (B).

The comparison of the results provided in Fig. 5(i-iv) validates the possible advantages that can be obtained by a communication among the two inverters for the feedforward compensation of their mutual couplings.

To asses the effectiveness of the proposed communication strategy in a realistic double six-phase drive, the simulation has been repeated maintaining the optimum feed forward compensation (iv) and introducing a delay, and related down sampling, of the transfer of the data among the inverters. The rate of the data transmission is reduced from $30 \mathrm{kHz}$, which is three times the switching frequency $\left(f_{s w}=10 \mathrm{kHz}\right)$, up to $62.5 \mathrm{~Hz}$. Considering the simulated operation of the drive, Fig. 5 shows, at the right hand side, the effect of a delay in the communication between the two controllers. At about 250-500 Hz communication frequency the advantages of the mutual coupling compensation result in performance similar to the one obtained without it, as Fig. 5(ii), and for a lower communication bandwidth (below $200 \mathrm{~Hz}$ ) it is 
clear that the compensation of the mutual couplings is inconvenient in the simulated scenario. However, the performance of the proposed feedforward compensations is almost unchanged up to $2 \mathrm{kHz}$ data rate, validating the possibility of using the proposed control strategy for improving the control of dual threephase drives with independent inverters by means of a relatively low bandwidth communication among the two control systems.

\section{Conclusions}

A new robust control algorithm for a double three-phase SPM machine has been proposed. The control is based on the assumption of having the two three-phase inverters independently controlled. The equations of the model have been presented in order to highlight the approximations that are considered while defining an independent control of the two converters.

A tuning methodology is proposed and compared with other existing techniques, showing the differences in terms of closed loop response of the control plants and related stability. The choice of the tuning strategy has been selected also on the basis of suitable feedforward compensations of the mutual couplings among the two sets of windings. Finally, a reliable communication among the two inverter controllers has been implemented, based on the compensation of the mutual bemfs of the systems, and has been validated by numerical simulations considering the effects of a low bandwidth communication.

The simulation results show that the proposed communication helps for a higher performance in the dynamic of the control, making the new methodology a possible solution for the management of double three-phase drives with independent inverters.

\section{References}

[1] F. Barrero and M. J. Duran: Recent advances in the design, modeling, and control of multiphase machines part i, IEEE Transactions on Industrial Electronics, vol. 63, no. 1, pp. 449-458, Jan 2016.

[2] M. J. Duran and F. Barrero: Recent advances in the design, modeling, and control of multiphase machines part ii, IEEE Transactions on Industrial Electronics, vol. 63, no. 1, pp. 459-468, Jan 2016.

[3] Y. Hu, Z. Q. Zhu, and M. Odavic: Comparison of two-individual current control and vector space decomposition control for dual three-phase pmsm, IEEE Transactions on Industry Applications, vol. 53, no. 5, pp. 4483-4492, Sep. 2017.

[4] E. Levi, R. Bojoi, F. Profumo, H. A. Toliyat, and S. Williamson: Multiphase induction motor drives - a technology status review, IET Electric Power Applications, vol. 1, no. 4, pp. 489-516, July 2007.

[5] I. Zoric, M. Jones, and E. Levi: Arbitrary power sharing among three-phase winding sets of multiphase machines, IEEE Transactions on Industrial Electronics, vol. 65, no. 2, pp. 1128-1139, Feb 2018.

[6] G. Sala, G. Valente, A. Formentini, L. Papini, D. Gerada, P. Zanchetta, A. Tani, and C. Gerada: Space vectors and pseudoinverse matrix methods for the radial force control in bearingless multisector permanent magnet machines, IEEE Transactions on Industrial Electronics, vol. 65, no. 9, pp. 6912-6922, Sep. 2018.

[7] E. Prieto-Araujo, A. Junyent-Ferr, D. Lavrnia-Ferrer, and O. Gomis-Bellmunt: Decentralized control of a nine-phase permanent magnet generator for offshore wind turbines, IEEE Transactions on Energy Conversion, vol. 30, no. 3, pp. 1103-1112, Sep. 2015.

[8] A. Galassini, A. Costabeber, M. Degano, C. Gerada, A. Tessarolo, and R. Menis: Enhanced power sharing transient with droop controllers for multithree-phase synchronous electrical machines, IEEE Transactions on Industrial Electronics, pp. 1-1, 2018.

[9] Yifan Zhao and T. A. Lipo: Space vector pwm control of dual three-phase induction machine using vector space decomposition, IEEE Transactions on Industry Applications, vol. 31, no. 5, pp. 1100-1109, Sep. 1995.

[10] H. S. Che, E. Levi, M. Jones, W. Hew, and N. A. Rahim: Current control methods for an asymmetrical six-phase induction motor drive, IEEE Transactions on Power Electronics, vol. 29, no. 1, pp. 407-417, Jan 2014.

[11] G. Sala, D. Gerada, C. Gerada, and A. Tani: Radial force control for triple three-phase sectored spm machines. part i: Machine model, in 2017 IEEE Workshop on Electrical Machines Design, Control and Diagnosis (WEMDCD), April 2017, pp. 193-198.

[12] S. Siala, E. Guette, and J. L. Pouliquen: Multi-inverter pwm control: a new generation drives for cruise ship electric propulsion, in European Power Electronics Conference (EPE2003), Toulouse (France), CDROM, Sep. 2003.

[13] G. Sala, P. Girardini, M. Mengoni, L. Zarri, A. Tani, and G. Serra: Comparison of fault tolerant control techniques for quadruple three-phase induction machines under open-circuit fault, in 2017 IEEE 11th International Symposium on Diagnostics for Electrical Machines, Power Electronics and Drives (SDEMPED), Aug 2017, pp. 213-219. 